\title{
The I-V Characteristics of $\mathrm{M}-\mathrm{Ba}_{x} \mathrm{Sr}_{1-x} \mathrm{TiO}_{3}-\mathrm{M}$ Thin Film Structures with Oxygen Vacancies. Part 1
}

\author{
V.V. Buniatyan*, H.R. Dastoyan \\ National Polytechnic University of Armenia (NPUA), 105 Teryan St., 0009, Yerevan, Armenia \\ *Corresponding author. Tel.+3 74913116 39.E-mail: vbuniat@seua.am, vbuniat@yahoo.com
}

\begin{abstract}
The paper presents a detailed study of the $I-V$ characteristics of $m$-ferroelectric $-m\left\{\mathrm{Pt} / \mathrm{Ba}_{x} \mathrm{St}_{1-x} \mathrm{TiO}_{3} / \mathrm{Pt}(\mathrm{Pt} / \mathrm{BST} / \mathrm{Pt})\right\}$ thin film structure. The Schottky barrier thermal/field assisted and Pool-Frenkel (PF) emission along with the punch-through in the central region of the film are proposed for the interpretation of experimental $I-V$ dependence. It is shown that both the Schottky barrier thermal/-field assisted emission and Pool-Frenkel emission from the oxygen vacancies conditioned traps may take place simultaneously. The effect of electric field on the electron (hole) emission from the trapping centers, in the symmetric Pt/BST/Pt thin film structure has been considered. The analysis of the PF effect indicates that the trapping centers are activated at very high electric fields, exceeding $10^{5} \mathrm{~V} / \mathrm{cm}$. In Part 2 of the paper, based on the results and assumptions pointed in Part 1, analytical expressions were derived for Schottky barrier thermal/-field assisted and Pool-Frenkel emission currents. The computer modeling theoretical dependencies of the $I-V$ characteristics has been compared with the experimental measured results and obtained good agreements.
\end{abstract}

\section{Keywords}

Ferroelectric; oxygen vacancy; Pool-Frenkel emission; Schottky barrier; trapping center.

(C) V.V. Buniatyan, H.R. Dastoyan, 2020

\section{Introduction}

The $I-V$ characteristics (leakage current) of thin ferroelectric film devices (non-volatile memory cell, varactors, micro-electromechanical systems (MEMS), ferroelectric FETs, phase and frequency agile microwave devices, etc.) has been a subject for extensive studies [1-23]. The main task of these investigations is understand and proposes methods for the reduction of the negative impact of the leakage currents on the device performance, and sometimes the $I-V$ characteristics themselves are used as a sensitive diagnostic tool for monitoring material parameters, such as concentration and distribution of defects and quality of interfaces $[11,16,23]$. The main experimental results reported for BST and PZT indicated a strong correlation of the leakage current with crystalline structure of the film [24-26]. The magnitude of the leakage current and the shape of the $I-V$ curves depend on the conduction mechanism on dielectric process in the ferroelectric and on the difference in the work function between the electrode and ferroelectric, nature and density of the interface states. The Poole-Frenkel emission [3, 4, 6, 15, 17-19, 27-30], the Schottky emission $[1-5,9,16,17,23,30]$, tunneling (field) emission, space-charge limited (SCL) regime $[1-4,9$, $16,23,30]$, ionic conductance, hopping conductance etc. have been considered. They are characterized by their voltage and temperature dependencies:

$$
\begin{gathered}
T^{2} \exp \left(\beta V^{\frac{1}{2}} T^{-\frac{1}{2}}-\frac{q \Phi}{k T}\right) ; \quad V \exp \left(2 \beta V^{\frac{1}{2}} T^{-\frac{1}{2}}-\frac{q \Phi}{k T}\right) ; \\
V^{2} \exp \left(-\frac{Z}{V}\right) ; \quad a\left[\frac{V}{d}+\beta\left(\frac{V}{d}\right)^{2}\right],
\end{gathered}
$$

where $k$ is the Boltzmann's constant, $T$ is the absolute temperature, $q$ is the electron charge. In general, it is not easy to distinguish between these mechanisms.

Typically, at lower fields and temperatures $(300 \mathrm{~K}$ and $\left.10^{4}-10^{5} \mathrm{~V} / \mathrm{cm}\right)$, the Schottky emission dominates 
[18], while at high fields $\left(10^{6} \mathrm{~V} / \mathrm{cm}\right)$ and high temperatures the Poole-Frenkel emission becomes dominant. The Schottky emission and space - charge limited current are not independent [1,2]. Currently it is well established that in ferroelectric thin films oxygen vacancies play a major role. The oxygen concentration is not a constant throughout of the film. It decreases sharply near the metal (for example, Pt) electrodes [2], approximately $50 \%$ of its value in the center $(\sim 20 \mathrm{~nm}$ from the metal surface). This oxygen deficient region in the film may have $n$-type conductivity in contrast to the $p$-type in the bulk of the film, where the density of the oxygen vacancies is not high. In oxide perovskite ferroelectrics there are three oxygen ions per unit cell. The density of the oxygen ions in the bulk of the film is $1.5 \times 10^{28} \mathrm{~m}^{3}[1,2,29$, $31,32]$, while in the $20 \mathrm{~nm}$ thick $50 \%$ depleted (by oxygen) interface layer it is $\left(3 \times 10^{20} / \mathrm{m}^{2}\right)$. The missing oxygen results in an image charge on metal electrode. The surface density of the changes on Pt electrode is $3 \times 10^{16}$ oxygen $/ \mathrm{cm}^{2}$ [2]. However, not all of the oxygen vacancies trap electrons. The increased density of the oxygen vacancies at the interface results in a dipole layer - lowering the electrical field in the film (due to increase of the voltage drop over the Pt/BST interfaces). The non-uniform distribution of the oxygen vacancies near the interfaces causes bending of the energy bands and changes the shape of the barrier, making it for charges easier to overcome. On the other hand, the experimentally observed leakage currents may be explained by conductivity associated with the oxygen vacancies $[1,10-16,25]$. The oxygen vacancies are the most mobile in perovskite ferroelectrics $[1,11$, $16,23,31]$. The experimental results of both mono crystalline and polycrystalline BST at high temperatures [29] shows that the conductivity of undoped polycrystalline titanate oxides depend on oxygen partial pressure of the ambient gas. At sufficiently high temperatures, the oxygen vacancies are double ionized, each supplying two electrons to the conduction band. This process at low oxygen partial pressure can be described as [21, 29]:

$$
\mathrm{O}_{0} \leftrightarrow \frac{1}{2} \mathrm{O}_{2}(\text { gas })+V_{0}^{\prime \prime}+2 e^{-}
$$

Oxygen vacancies act as donors [5, 7, 11-16, 30, $33,37,40]$ causing $n$-type conductivity. Moreover, depending on the density of the oxygen vacancies and the density of the background impurities the conductivity may change from $n$-to $p$-type $[1,11,16$, 23]. It is assumed that the donor state is strongly localized around the nearest titanium ions. In the neutral state the donor level is double occupied, and there is a reduced repulsive interaction between the vacancy and neighboring cations. The interfacial vacancies cause distortion of the crystal lattice and polarization fields around the vacancy. This makes the levels deeper and causes them to act as charge traps $[33,34]$. The interfacial built - in electric fields associated with the trapping centers and oxygen vacancies results in changes the interfacial permittivity of the films $[17,34]$. It is worthwhile to notice that the oxygen vacancies are not to be only main defect of ferroelectric films $[2,34]$. The $\mathrm{Ba}, \mathrm{Sr}$ vacancies in BST result in shallow acceptor levels [2, 21, 23, 25, 33-35]. Dopants also result in levels in the forbidden band. $\mathrm{Nb}$ gives a shallow level [35], while $\mathrm{Mn}, \mathrm{Gr}$, and $\mathrm{Fe}$ give levels near midgap. Pt gives a deep level also near midgap $[35,36]$.

Most of metal-ferroelectric junctions lie between the Schottky and Bardeen limits. In $\mathrm{Pt}-\mathrm{SrTiO}_{3}$ contact $[2,3,5,37,38]$ the effect of vacancies on barrier height becomes important for the density of vacancies $10^{18}-10^{22} \mathrm{~cm}^{-3}[2,32,35]$. The, electrochemical interaction of the electrodes with carriers in ferroelectric may induce "dead layers" [11, 16, 23, 39]. In the case of interfacial surface states and/or dopants there may be a transfer of charge and the barrier height cannot be defined as a difference between the metal work function $\Phi_{m}$ and the insulator electron affinity $\chi$ $[5,16,30]$. In this case the resulting barrier height is $[5,12,13,16-20,26,40,41]$ :

$$
\Phi_{n} \cong S\left(\Phi_{m}-\Phi_{S}\right)+\left(\Phi_{S}-\chi\right)+S \Phi_{S} .
$$

The slop parameter $S$ is semiconductor (in the case ferroelectric) specific, with $S=0$ describing the Bardeen limit of strong pinning and $S=1$ describing the Schottky limit of weak pinning [17-19, 42]. $S$ and $\Phi_{S}$ (which is known as the charge neutrality level) are respectively 0.26 and $2.6 \mathrm{eV}$ (above the $E_{v}$ ) [17-19, 42]. The calculated barrier height for $\mathrm{SrTiO}_{3}$ on $\mathrm{Pt}$ is about $0.9 \mathrm{eV}$ [5], which is close to the $0.8 \mathrm{eV}$ found by photoemission [43] and the $1.1 \mathrm{eV}$ [3]. These low barrier heights are in agreement with the other reports $[3,5,6,12,13,15,17,38]$. In summary, for low 
density of oxygen vacancies the undoped ferroelectrics titanates films are considered to have slightly $p$-type conductivity due to the background impurities (i.e. $\left[\mathrm{Na}^{+}\right.$for $\mathrm{Pb}^{+2}, \mathrm{Fe}^{+3}$ for $\mathrm{Ti}^{+4}[21,44]$ ).

In reality, most of the ferroelectric films are rich in oxygen vacancies, especially at the interfacial with the electrodes regions $[1-3,6,11-16,21,23,30,33,34$, 39, 40, 45]. In fact, the Kelvin probe study [1, 3, 30, 33, 40, 45] proved that in $\mathrm{Pt} \mathrm{BaTiO}_{3}$ thin film structure the work function changes from $2.5 \pm 0.3$ for surfaces to $4.4 \pm 0.4 \mathrm{eV}$ the bulk of the material.

\section{The Proposed Model}

\section{of the thin film $\mathrm{Pt} / \mathrm{Ba}_{x} \mathrm{St}_{1-x} \mathrm{TiO}_{3} / \mathrm{Pt}$ structure}

The three layer model to be considered consists of metal electrodes, Fig. 1, n-type interfacial oxygen vacancy rich layers (uncompensated donor concentration $n_{d}$ with the thickness of $\delta_{1}$ ) with the homogeneous distribution of the vacancies, and a poor $p$-type ferroelectric film (core). It is assumed, that the concentration of the oxygen vacancies is large at the interfacial with the metal $(\mathrm{Pt})$ contact region, and that some of these vacancies trap electrons and create space charge regions. For the simplicity both metal/ferroelectric contacts assumed to be identical. It is also assumed that there are interfacial surface states between the metal contacts and $n$-type interfacial regions. The thickness these layers with the surface states is denoted by $\delta_{0}$. The density of the oxygen vacancies in the middle part of the ferroelectric film (core) is assumed to be relatively low.
The work function $(\sim 5.6 \mathrm{eV})$ of $\mathrm{Pt}$ is greater, than that of the ferroelectric film $(\sim 3.2 \mathrm{eV})$, which means that the currents will be emission limited. In equilibrium and at very low temperatures all of the trapped electrons (holes) would be in traps: at high temperatures and in presence of an applied field some of these electrons (holes) will be exited into shallow traps or conduction levels, either thermally or due to action of the field [1].

The $I-V$ characteristic of the proposed model is considered neglecting the diffusion currents associated with the charge concentration gradients. It is also assumed that electrons and holes flow either by PoolFrenkel emission (Schottky intrinsic emission) or by Schottky thermionic - field emission at high fields, when whole ferroelectric is in reach - through (flat band) condition [16-19]. In the present analysis the voltage applied to the structure is assumed to be greater than the voltage necessary to realize the punch-through condition. Additionally, a carrier released from a trap is expected to move in the electric field so, that the retrapping process may be neglected. Based on the above assumptions the condition current density $J$ in the $\mathrm{Pt} /$ ferroelectric/Pt structures is considered to be a sum of two contributions:

$$
J=J_{1}+J_{2}
$$

where $J_{1}$ is due to reach - through condition with Schottky field - emission; $J_{2}$ is due to field - enhanced thermal excitation of trapped electrons and holes into the condition and valence bands, which is known as the internal Schottky effect or the Poole-Frencel effect.

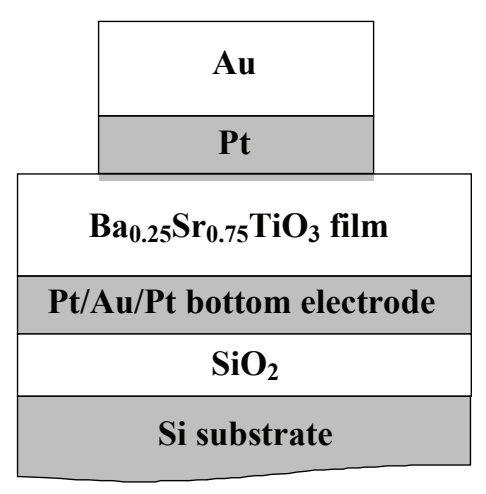

a)

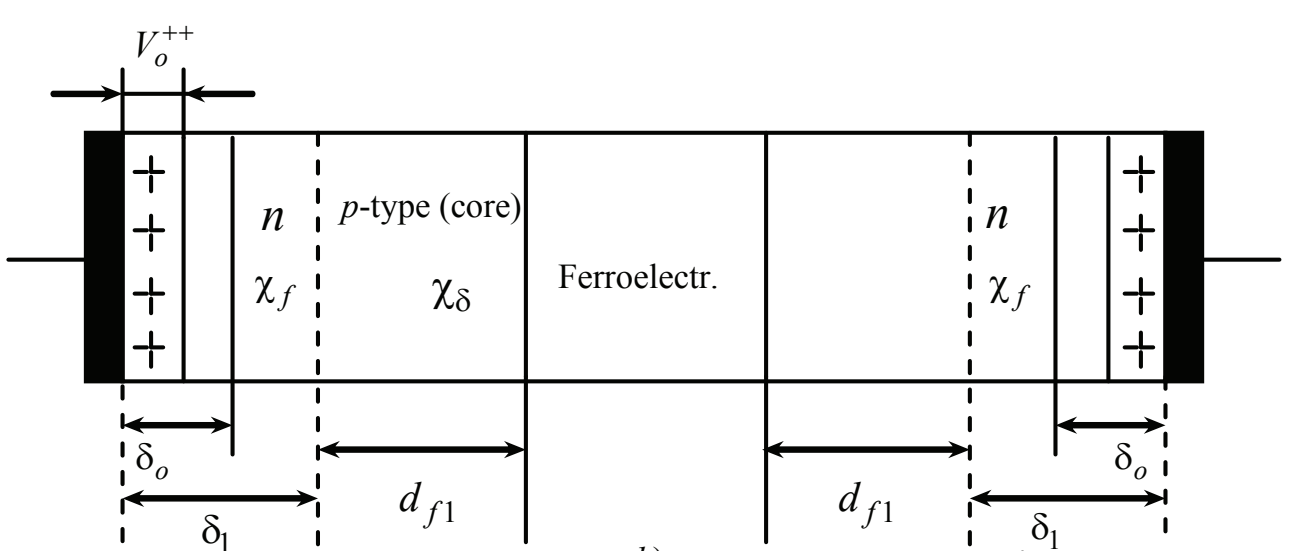

b)

Fig. 1. Cross section of $\mathrm{Pt}-\mathrm{Ba}_{0.25} \mathrm{Sr}_{0.75} \mathrm{TiO}_{3}-\mathrm{Pt}$ test structure (a) and 1D-model (b) 


\section{Model of the current mechanisms in $\mathrm{Pt} / \mathrm{Ba}_{x} \mathrm{St}_{1-x} \mathrm{TiO}_{3} / \mathrm{Pt}$ structure}

\section{The potential barrier}

Assume that concentration of oxygen vacancies is $n_{d}$. Each vacancy gives two electrons to conduction band. In equilibrium condition, a part of them are trapped in interfacial surface states (below Fermi level) in energy depth of $q \Phi_{0}$ (Fig. 2a) [17-19]. The others are trapped in corresponding levels, which is assumed to be presence in the band gap with the energy depth of $E_{t n}$. Assume that interfacial surface layer width is $\delta_{0}$ (about $5 \sim 10 \mathrm{~nm}$ ) [2]. If the trapped in the surface states and $\delta_{0}$ layer traps level electron concentration is $n_{t 0}^{\prime}\left(n_{t 0}^{\prime}>n_{t 0}\right)$, where $n_{t 0}$ is the concentration of captured electrons in the core), the fixed positive charge density will be formed with the concentration of $\rho_{v}^{+}$. The local polarization due to the non-compensated positive charge one can estimate by $[11,16,23]$ $P_{e} \sim \frac{\rho_{v}^{+} \delta_{0}}{3}$.

Now, it is assumed that fixed positive charge in the $\delta_{0}$ interfacial layers is $\rho^{+}$. From Poission's equation [17-19]:

$$
\frac{d E_{\delta}}{d x}=\frac{\rho_{\mathrm{v}}^{+}}{x_{\delta}},
$$

where $x_{\delta}$ is the dielectric constant of layer $\delta_{0}$ and $E_{\delta}$ is the electric field.

Integration of (1) gives:

$$
E_{\delta}(x)=C_{0}+\int_{0}^{x} \frac{\rho^{+}(x)}{x_{\delta}} d x .
$$

with the boundary condition: $x=\delta_{1}, E_{\delta}\left(\delta_{1}\right)=0$, (where $\delta_{1}$ is the width of the $n$-type interfacial space charge layer) one gets $C_{0}=-\int_{0}^{\delta_{1}} \frac{\rho^{+}(x)}{x_{\delta}} d x$, and:

$$
E_{\delta}(x)=-\int_{x}^{\delta_{1}} \frac{\rho^{+}(x)}{x_{\delta}} d x .
$$

The potential can now be obtained using

$$
E(x)=-\frac{d V(x)}{d x},
$$

and the entire potential drop is

$$
V(0)-V\left(\delta_{1}\right)=\int_{0}^{\delta_{1}}\left(-\int_{x}^{\delta_{1}} \frac{\rho^{+}}{x_{\delta}} d x\right) d x=-\int_{0}^{\delta_{1}} x \frac{\rho_{v}^{+}(x)}{x_{\delta}} d x,
$$

where the last expression was obtained from integration by parts. The result is

$$
V(0)-V\left(\delta_{1}\right)=\varphi_{K}=\int_{0}^{\delta_{1}} x \frac{\rho^{+}(x)}{x_{\delta}} d x .
$$

Integration of (2), assuming constant concentration of $\rho_{1}^{+}$for $x<\delta_{0}$, constant concentration $\rho_{v}^{+}$for $x>\delta_{0}$ $\left(\rho_{1}^{+}<\rho_{v}^{+}\right.$due to trapped surface states) and an appropriate Dirac $\delta$ function at the interface, one obtains

$$
\begin{gathered}
\varphi_{k}=\frac{1}{x_{\delta}}\left\{\int_{0}^{\delta_{0}^{-}} x \rho_{1}^{+} d x-\int_{\delta_{0}^{-}}^{\delta_{0}^{+}} x \sigma_{s} \delta\left(x-\delta_{0}\right) d x+\int_{\delta_{0}^{+}}^{\delta_{1}} x \rho_{v}^{+} d x\right\}= \\
=\frac{\rho_{1}^{+} \delta_{0}^{2}}{2 x_{\delta}}+\frac{\rho_{v}^{+}\left(\delta_{1}^{2}-\delta_{0}^{2}\right)}{2 x_{\delta}}-\frac{\delta_{0} \sigma_{s}^{+}}{x_{\delta}},
\end{gathered}
$$

where $\sigma_{s}^{+}$is the interface charge density $\left(\mathrm{cm}^{-2}\right)$

So,

$$
\varphi_{k}=\frac{\rho_{1}^{+} \delta_{0}^{2}}{2 x_{\delta}}+\frac{\rho_{v}^{+}\left(\delta_{1}^{2}-\delta_{0}^{2}\right)}{2 x_{\delta}}-\frac{\delta_{0} \sigma_{s}^{+}}{x_{\delta}} .
$$

Note, that for the Schottky junction $\rho_{1}^{+}=\rho_{v}^{+}, \sigma_{s}^{+}=0$.

Solving the Eq. (3) in regards to $\delta_{1}$, we can obtain:

$$
\delta_{1}=\sqrt{\frac{2 x_{\delta} \varphi_{k}^{\prime}}{\rho_{v}^{+}}}
$$

where $\varphi_{k}^{\prime}=\varphi_{k}+\frac{\rho_{1}^{+}}{2 x_{\delta}}\left(1-\frac{\rho_{1}^{+}}{\rho_{v}^{+}}\right)^{2} \delta_{0}+\frac{q \sigma_{s}^{+}}{x_{\delta}} \delta_{0}$.

If the polarization field in $\delta_{0}$ layer is taken into account, for the $\varphi_{k}$ and $\delta_{1}$, then:

$$
\varphi_{k}^{\prime \prime}=\varphi_{k}^{\prime} \pm \frac{P_{\delta} \delta_{0}}{x_{\delta}} ; \quad \delta_{1}=\sqrt{\frac{2 x_{\delta} \varphi_{k}^{\prime \prime}}{\rho_{v}^{+}}} .
$$

Estimation $\varphi_{k}$ for $\mathrm{SrTiO}_{3}$ will be considered as an example. If $x_{f}=300[7,11,16,22,23,41]$, $x_{\delta} \sim 0.01 x_{f} \quad\left(x_{f}\right.$ is the ferroelectric core dielectric constant), $n_{d} \sim 1.6 \cdot 10^{-18} \mathrm{~cm}^{-3}[2,5,11,17,25,30,32]$, assuming $\rho_{v}^{+} \sim n_{d}+n_{t_{0}}, \quad n_{t_{0}} \sim 0.25 n_{d}, \quad \delta_{0} \cong 0.2 \mathrm{~nm}$, 


\section{AM\&T}

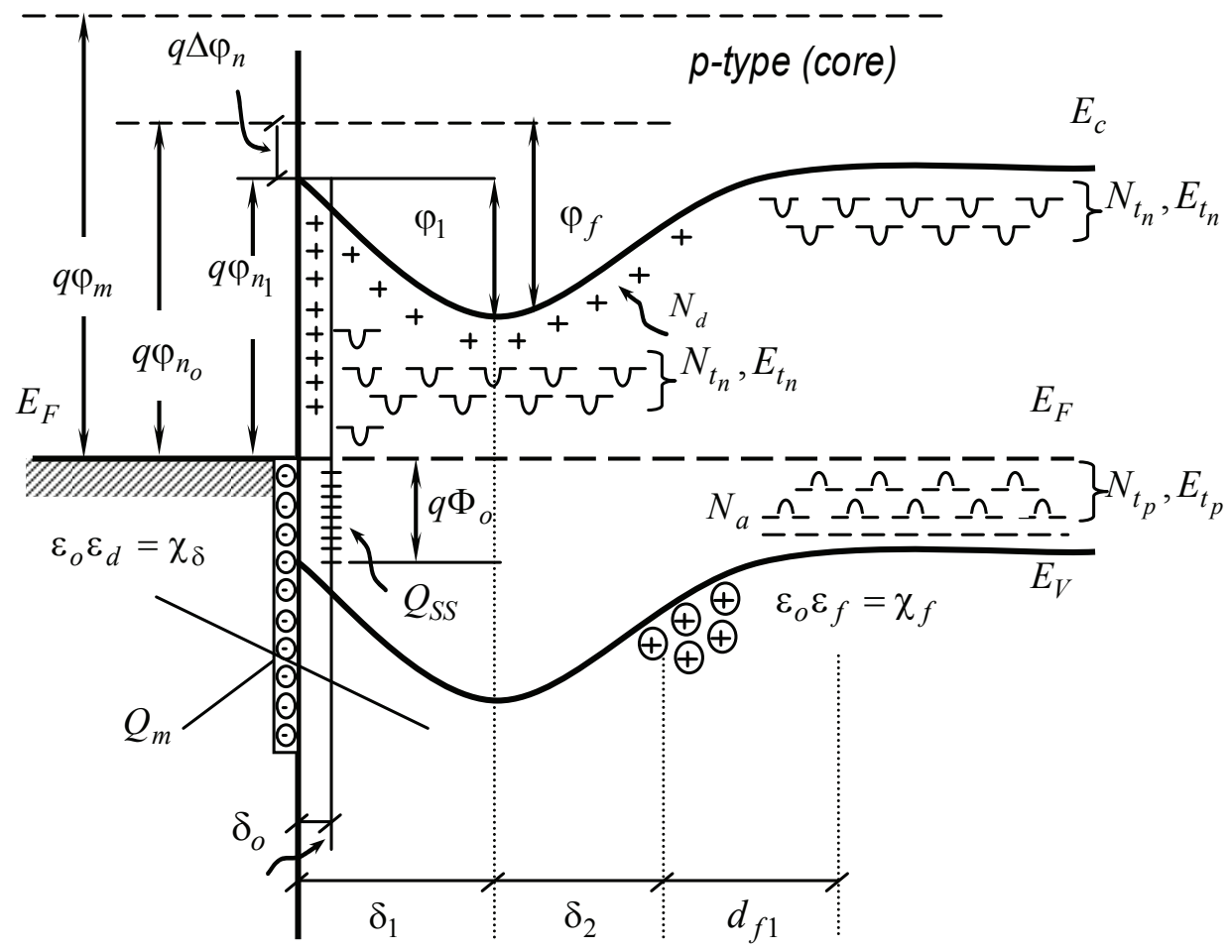

a)

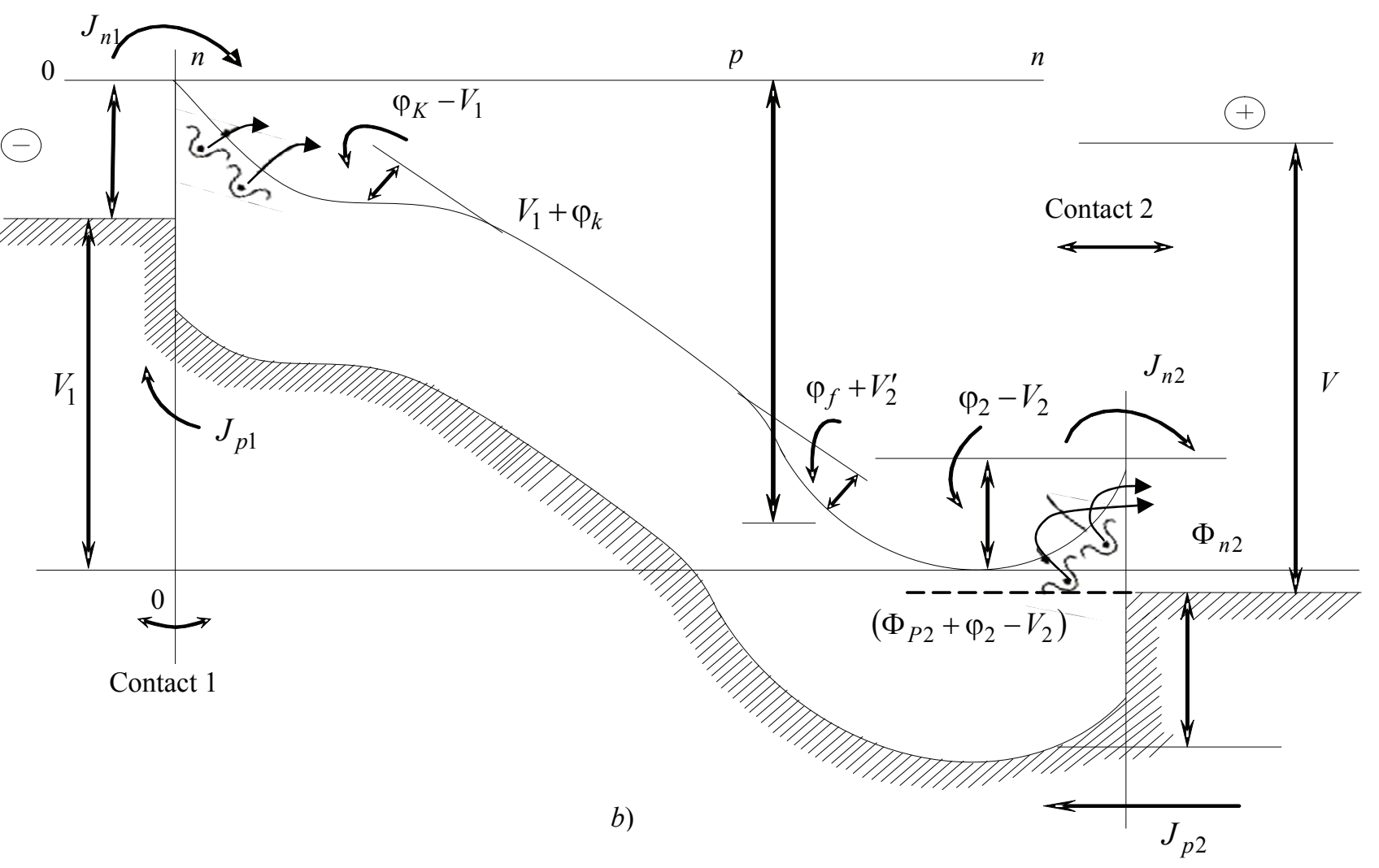

Fig. 2. Potential profile of MFM structure are thermal equilibrium (a) and under bias (b) (with positive bias contact 2) 
$\rho_{1}^{+} \cong 0.75 \rho_{v}^{+}[1,3,11,16,17-19], \delta_{1}=20 \mathrm{~nm}$ [2], one can obtain $\varphi_{k} \cong 0.7 \mathrm{eV}$, without taken into account polarization effect, which coincides with result, obtained in $[2,3,5,14-21,24,30,37,38,40,44,45]$. If $n_{d} \sim 10^{18} \mathrm{~cm}^{-3}, N_{a} \sim 10^{12} \mathrm{~cm}^{-3}, \varphi_{k} \approx k T \ln \left[\frac{N_{d} N_{a}}{n^{2}}\right]$, $\varphi_{k}=1.1 \mathrm{eV}$. This result indicates that this is not an accurate way to estimate the built-in potential in metal/ferroelectric contacts. The result $\varphi_{k}=1.1 \mathrm{eV}$ is larger than the barrier height, in contradiction to many experimental and calculated results $[2,3,21,24,25,37$, 38]. Additionally, the estimation of $\varphi_{k}$ in this simple way is not correct since it does not take into account the interface states and their effect of barrier height and therefore the built-in potential. It is believed that more realistic is $[2-6,10,15,23,26,41]: q \Phi_{n} \approx(0.8 \div 0.9) \mathrm{eV}$, $\varphi_{k} \approx(0.6 \div 0.7) \mathrm{eV}$. For calculation of ferroelectricmetal barrier height the method, described in [36] will be used, assuming surface states in interfacial region. The density of the charge in the surface states is given by

$$
\rho_{s S}^{-}=q D_{s}\left(E_{g}-q \Phi_{0}-q \Phi_{n}-q \Delta \Phi\right),
$$

where $D_{s}$ is the surface state density between $q \Phi_{0}$ and Fermi level (tapped electrons), $\Delta \Phi$ is the Schottky barrier lowering due to image force:

$$
\Delta \Phi=\sqrt{\frac{q\left(E_{m}-E_{p}\right)}{4 \pi \varepsilon_{0}}},
$$

where $E_{m}$ is the applied field and $E_{i}$ is built-in field due to polarization [16-19, 23, 30, 33, 37]. The surface charge density in the $\delta_{1}$ depletion layer will be given by:

$$
\rho_{s c}^{+}\left(\delta_{1}\right)=\sqrt{2 x_{\delta} \rho_{v}^{+}\left(\Phi_{n}-V_{n}+\Delta \Phi-\frac{k T}{q}\right)},
$$

where $V_{n}$ is the voltage which is drop in the metalferroelectric contact $\left(\delta_{1}\right.$-contact), $\Phi_{n}$ is the barrier height, $k T / q$ is due to free charge, which will be neglected. So, in $\delta_{1}$ layer the resulting surface charge density is the sum of $\rho_{s s}^{-}\left(\delta_{1}\right)$ and $\rho_{s c}^{+}\left(\delta_{0}\right)$. Consequently, the surface density of the negative charge in metal is given by [19]:

$$
Q_{m}=-\left(\mid \rho_{s s}^{-}\left(\delta_{1}\right)-\rho_{s c}^{+}\left(\delta_{1}\right)\right),
$$

By using the Gauss's law [19], one finally obtains:

$$
q \Phi_{n}=\frac{q \Phi_{m}-q \chi-q \Delta \Phi\left(1+c_{1}\right)+c_{1}\left[E_{g}-q \Phi_{0}\right]}{1+c_{1}},
$$

where $c_{1}=\frac{q^{2} D_{s} \delta_{0}}{\chi_{\delta}}$

Assume, that $\delta_{0} \sim(1.6 \div 2) \AA[17-19], c_{1}<<1$ :

$$
q \Phi_{n}=q \Phi_{m}-q \chi-q \Delta \Phi \text {. }
$$

If $q \Phi_{0} \sim 0.3 \mathrm{eV}$ [19], $E_{g}=3.3 \mathrm{eV}, \chi=4.1 \mathrm{eV}$, $q \Phi_{m} \approx 5.3 \mathrm{eV}, q \Phi_{n} \approx 0.9 \mathrm{eV}$.

The estimation of the barrier height with the pinning parameter $[5,11,15,17-19,42]$, characterizing the contact pinning properties, for the $q \Phi_{n}$ gives $q \Phi_{n} \approx 0.89 \mathrm{eV}$ with the $S \sim 0.35$, charge neutrality level $q \Phi_{s} \approx 4.7 \mathrm{eV}$ below the vacuum level $[5,18,19,21,30$, 41-44]. It is necessary to note, that the parameter, described in $[5,18,19,30,41]$ is $S=\left(1+\frac{q^{2} N \delta}{\varepsilon_{i} \varepsilon_{0}}\right)^{-1}$, where $N$ is the area density of the surface states and $\delta$ is the there extent into insulator. As it is evident from the expression for $c_{1}$ and $S, c_{1}=S$. If $\varepsilon_{i} \varepsilon_{0}=\chi_{d}=5 \varepsilon$, $S=0.35, \delta \approx 2 \AA$, one may estimate the $D_{S}=N$, which is given $D_{s}=1.6 \cdot 10^{13} \frac{\text { state }}{\mathrm{cm}^{2} \cdot \mathrm{eV}}$.

For the calculation of the $I-V$ characteristics and built-in potential between interfacial $n$-type layer $\left(\delta_{1}\right)$ and $p$-type (core) ferroelectric film, we will be based on following:

i) as it is noted in the introduction the core of the ferroelectric film has poor p-type conductivity. It means that in some plane from the metal contact a transition from $n$-type to $p$-type conductivity takes place;

ii) the interaction of electrodes with charge carriers in the ferroelectric may result in so-called space charge "depletion" effect $[11,16,17,23,30]$ i.e. the removal of the carriers from the narrow regions near the electrodes, so that space charged regions of the ionized impurities are formed near the electrodes. For a wide gap and heavily compensated ferroelectric, this build-in charge is related to deep trapping centers and oxygen vacancies;

iii) if there is take place the change of type of electrical conductivity and formation of space charge near the metal contacts, it means, that $d_{f}$ width of space 
layer of ferroelectric can to be less as $\mathrm{h}$, where $\mathrm{h}$ is the thickness of film $[11,23,30]$;

iii) as the applied voltage of ferroelectric films usually is very large, it is reasonable to assume that the core of film is in fully depleted.

Based on this assumption we will use the theory of $I-V$ characteristics MSM, MIS structures developed in [17-19].

\section{The Pt/BST/Pt structure under external DC bias}

When an external DC bias is applied to $\mathrm{Pt} / \mathrm{BST} / \mathrm{Pt}$ structure, as shown in Fig. $2 b$, the barriers $\Phi_{n 1}$ and $\varphi_{t 2}$ are reverse biased, the barrier $\varphi_{t 1}$ and $\Phi_{n 2}$ are forward biased. The applied voltage is shared between two contacts and ferroelectric film, so that

$$
V_{e}=V_{1}+V_{2}+V_{3} \text {. }
$$

The reverse current density $J_{n 1}$ for the contact (1) is given by [17-19]:

$$
\begin{gathered}
J_{n 1}=A_{n}^{*} T^{2} \exp \left(-\beta \varphi_{n 1}\right) \times \\
\times \exp \left[\beta\left(\Delta \Phi_{n 1}+\alpha_{1} E_{m 1}\right)\left[1-\exp \left(-\beta V_{1}\right)\right]\right],
\end{gathered}
$$

where $\beta=\frac{q}{k T}, E_{m 1}$ is the maximum electric field (at $x=0$ ), $\alpha_{1}$ is the intrinsic barrier lowering coefficient $[17-19,42]$, and $\Delta \Phi_{n 1}$ is the Schottky barrier lowering:

$$
\Delta \Phi_{n 1}=\sqrt{\frac{q E_{m 1}}{4 \pi \chi_{\delta}}-\frac{P_{\delta} \delta_{0}}{\chi_{\delta}}}
$$

with $E_{m 1}=\sqrt{\frac{4 q\left(n_{d}-n_{t o}\right)\left(V_{1}+\varphi_{k}\right)}{\chi_{\delta}}}$.

In Fig. $2 b \Phi_{n 1}$ and $\Phi_{n 2}$ are the electron barrier heights, $\varphi_{1}$ and $\varphi_{2}$, are the built-in potentials for contacts 1 and 2 with the metal $/ n$-type interfacial ferroelectrics respectively, $\varphi_{f}$ is the built-in potential for $n$-type interfacial ferroelectric and $p$-type core ferroelectric, $V_{1}$ and $V_{2}$ are the voltage drops in the contacts 1 and 2 respectively, $\Delta \Phi_{n}$ is the image force lowering of $\Phi_{n 1}, \delta_{1}$ is the width of the space charge between metal $/ n$ interfacial region, $\delta_{2}$ is the space charge layer-width between $n / p$-core ferroelectric region, $d_{f 1}$ is the depletion layer width of $p$-type ferroelectric, $Q_{m}$ is the surface charge density on metal, $Q_{s s}$ is the surface - state density on $n$-type interfacial region, $\delta_{o}$ is the thickness of interfacial layer, $\chi_{d}$ is the dielectric constant of interfacial layer, $\chi_{f}$ is the dielectric constant of ferroelectric core, $\Phi_{o}$ is the energy levels at surface, $\Phi_{n 0}$ value of $\Phi_{n 1}$ at zero electric field, $\Phi_{m}$ is the work function of metal, $N_{t n}, N_{t p}$ are the traps for electrons and holes, respectively, $E_{t n}$ and $E_{t p}$ are the traps energy depths.

The forward current density $J_{n 2}$ is given by

$$
\begin{gathered}
J_{n 2}=A_{n}^{*} T^{2} \exp \left(-\beta \varphi_{n 2}\right) \times \\
\times \exp \left[\beta\left(\Delta \Phi_{n 2}+\alpha_{2} E_{m 2}\right)\left[\exp \left(\beta V_{2}-1\right)\right]\right],
\end{gathered}
$$

where

$$
\begin{gathered}
\Delta \Phi_{n 2}=\sqrt{\frac{q E_{m 2}}{4 \pi \chi_{\delta}}-\frac{P_{\delta} \delta_{0}}{\chi_{\delta}}} ; \\
E_{m 2}=\sqrt{\frac{4 q\left(n_{d}-n_{t o}\right)\left(\varphi_{k}-V_{2}\right)}{\chi_{\delta}}} .
\end{gathered}
$$

From current continuity requirements, assuming symmetric contacts $\left(J_{n 1}=J_{n 2}, \quad \Phi_{n 1}=\Phi_{n 2}, \quad \alpha_{1}=\alpha_{2}\right.$, $\left.\varphi_{k 1}=\varphi_{k 2}\right)$, the relationship between $V_{1}$ and $V_{2}$ is:

$$
\begin{gathered}
{\left[\frac{q^{3}\left(n_{d}-n_{t o}\right)}{8 \pi^{2} \chi_{\delta}^{3}}\right]^{\frac{1}{4}}\left[\left(V_{1}+\varphi_{k}\right)^{\frac{1}{4}}-\left(\varphi_{k}-V_{2}\right)^{\frac{1}{4}}\right]=} \\
=\frac{1}{\beta} \ln \left[\frac{\left(\mathrm{e}^{\beta V_{2}}-1\right)}{\left(1-\mathrm{e}^{\beta V_{1}}\right)}\right] .
\end{gathered}
$$

Assume that the thickness of the ferroelectric film is larger than that of the space charge (depletion) widths of $d_{f 1}+d_{f 2}$. Then the reach-through voltage is:

$$
V_{R T}=V_{F B}-2 h \gamma \delta_{2},
$$

where $V_{F B}=\left(h^{2} / \xi^{2}\right) \gamma$ is the flat-band voltage, which makes $V_{2}=\varphi_{k}, \xi=1+\frac{n_{d}-n_{t o}}{N_{t p}-P_{t o}}, P_{t o}$ is the concentration of the captured holes, $\gamma=\frac{1}{a}-\frac{(\xi-1)^{2}}{b}, a=\frac{2 \chi_{\delta}}{q\left[N_{d}-n_{t o}\right]}=$ $=\frac{2 \chi_{\delta}}{q n_{c}}, b=\frac{2 \chi_{f}}{q\left(N_{t p}-P_{t o}\right)}=\frac{2 \chi_{f}}{q P_{c}}, n_{c}$ and $P_{c}$ are the free electron and holes concentration in the conduction (valence) band of the ferroelectric when an external field is applied:

$$
n_{c} \cong \frac{N_{c}}{N_{t n}}\left[\mathrm{e}^{-\frac{E_{F}}{k T}}+\mathrm{e}^{-\frac{E_{t n}}{k T}}\right]
$$




$$
p_{c} \cong \frac{N_{v}}{N_{t p}}\left[\mathrm{e}^{-\frac{E_{F}}{k T}}+\mathrm{e}^{-\frac{E_{t p}}{k T}}\right]
$$

$N_{c}$ and $N_{v}$ are the conductance and valence bands effective densities of states, $N_{t n}$ and $N_{t p}$ are the concentrations of electron and hole traps, respectively, $h$ is the thickness of the ferroelectric film, and $\delta_{2}=\sqrt{a\left(\varphi_{k}-V_{2}\right)}$.

After reach-through [17-19], Fig. 3, the electric field vary linearly from $x=0$ to $x=h$. The magnitudes of the fields at $x=0$ and $x=h$, are:

$$
\begin{aligned}
& E_{m 1}=\frac{\left(V+V_{F B}+2 \Delta \varphi\right)}{h}, \\
& E_{m 2}=\frac{\left|V-V_{F B}\right|}{h},
\end{aligned}
$$

$\Delta \varphi=\varphi_{k 1}-\varphi_{k 2}=0$ for the symmetrical structure. The field will pass through zero at a certain position (near to the (2) contact) $x_{0}$ :

$$
x_{0}=\frac{\chi_{f} E_{m 1}}{q\left[N_{t p}-P_{t o}\right]}=\frac{\chi_{f} E_{m 1}}{q P_{c}} .
$$

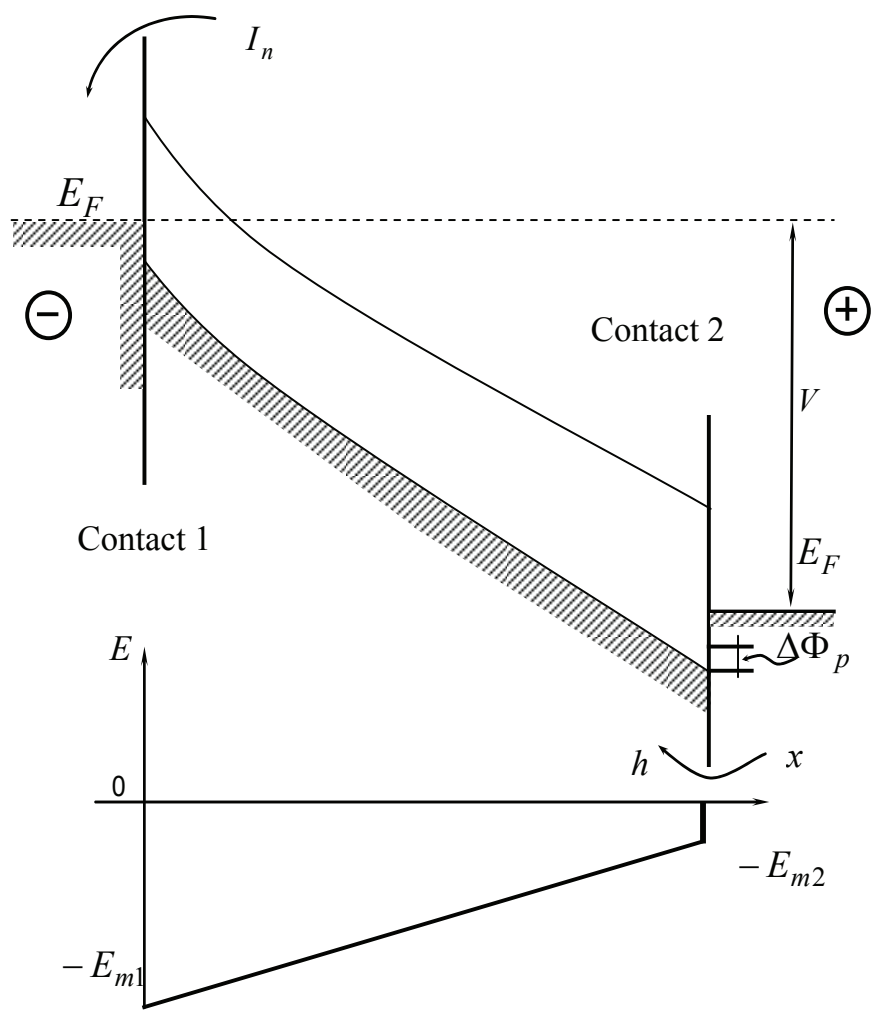

Fig. 3. Condition of flat-band at which the energy band at $\boldsymbol{x}=\boldsymbol{h}$ becomes flat, $\Delta \varphi_{p 2}$ is the image force lowering of $\varphi_{p 2}$
The voltages in the contacts (1) and (2) are given by $\Delta \varphi_{p 2}: V_{1}+\varphi_{k}=\frac{E_{m 1} X_{0}}{2}, \varphi_{k}-V_{2}=\frac{E_{m 2}\left(h-X_{o}\right)}{2}$, $V_{1}=\frac{\chi_{f}}{2 q P_{c}} \frac{\left(V+V_{F B}\right)^{2}}{h^{2}}, V_{2}=\varphi_{k}-\frac{\left\{h-X_{o}\right\}}{2} \frac{\left|V-V_{F B}\right|}{h}$.

Since the space charge (depletion) edge of the reverse - biased contact is pushed into that of the forward-biased one, the forward barrier is rapidly reduced with increasing bias. The electron current is now limited by the reverse biased contact and is given by:

$$
J_{n 1}=A_{n}^{*} T^{2} \mathrm{e}^{-\beta \Phi_{n_{1}}} \mathrm{e}^{\beta\left(\Delta \Phi_{n_{1}}+\alpha E_{m_{1}}\right)}\left(1-\mathrm{e}^{-\beta V_{1}}\right),
$$

where $E_{m 1}=\frac{\left(V+V_{F B}\right)}{h}$.

The maximum voltage that can be applied to $\mathrm{Pt} / \mathrm{BST} / \mathrm{Pt}$ structure is determined by the breakdown voltage. Given the strong dependence of ionization rates on field, and the trapped holes at the interface and core regions of the BST film, it is reasonable to assume, that at certain bias field and concentration of filled traps, the current may be governed by PoolFrenkel [17-19, 42] mechanism. The breakdown voltage is found from (7). For $E_{m 1}=E_{B}$, $V_{B} \cong E_{B} h-V_{F B}$.

\section{Acknowledgments}

The study was supported by RA MESCS Science Committee as part of the research project No. 19YR-2J050.

\section{References}

1. O'Dwer J.J. Current-Voltage Characteristics of Dielectric Films. J. Appl. Phys., 1966, 37(2), 599-601.

2. Scott J.F., Aranjo C.A., Melnick B. M. et al. Quantitative measurement of space-charge effects in lead ziconate-titanate memories. J. Appl. Phys., 1991, 70(70), 382-388.

3. Dietz G.W., Antpohler W., Klee M., et al. Electrode influence on the charge transport through $\mathrm{SrTiO}_{3}$ thin films. J. Appl. Phys., 1995, 78, 6113-6118.

4. Pulfrey D.L., Shousha A.H.M., and Young L. Electronic Conduction and Space Charge in Amorphous Insulating Films. J. Appl. Phys., 1970, 41, 2838-2834.

5. Robertson J. and Chen C.W. Schottki barrier heights of tantalum oxide, barium strontium titanate, lead titanate, and strontium bismuth titanate. J. Appl. Phys., 1999, 74(8), 1168-1170. 
6. Pintilie L., Boerasu I., Gomes M.J.M. et al. Metal-ferroelecric-metal structures with Schottky contacts. Analysis of the experimental current-voltage and capacitance-voltage characteristics of $\mathrm{Pb}(\mathrm{Zn}, \mathrm{Ti}) \mathrm{O}_{3}$ thin films. J. of Applied Physics, 2005, 98, 124104-124104.

7. Baniecki J.D., Laibonitz R. B., Shaw T.M., et al. Hidrogen induced tunnel emission in $\mathrm{Pt} /\left(\mathrm{Ba}_{x} \mathrm{Sr}_{1-x}\right) \mathrm{Ti}_{1+y} \mathrm{O}_{3+z} / \mathrm{Pt}$ thin films Capacitors. Appl. Phys., 2001, 89, 2873-2885.

8. Hwang C.S, Lee B.T., Kang C.S. et al. Depletion layer thickness and Schottky type carrier injection at the interface between $\mathrm{Pt}$ electrodes and $(\mathrm{Ba}, \mathrm{Sr}) \mathrm{TiO}_{3}(\mathrm{Ba}, \mathrm{Sr}) \mathrm{TiO}_{3}$ thin films. J. of Applied Physics, 1999, 85, 287-292.

9. Frank R.I., Simmons J.G. Space-charge effects on emission-limited current flow in insulators. J. Appl. Physics, 1967, 38, 832-840.

10. Saha S., Kaufman D.X., Streiffer S.K. et al. Anomalous leakage current characteristics of $\left.\mathrm{Pt} /\left(\mathrm{Ba}_{0.75} \mathrm{Sr}_{0.25}\right) \mathrm{Ti}_{1+y} \mathrm{O}_{3+z} / \mathrm{Pt}\right)$ thin films grown by metal organic chemical vapor deposition. J. Appl. Phys., 2003, 28(24), 3866-3868.

11. Tagantsev A.K., Sherman V.O., Astafiev K.F. et al. Ferroelectric Materials for Microwave Tunable applications. J. of Electroceramics, 2003, 11, 5-66.

12. Wang Y.-P., TsengT.-Y. Electronic defect and trap-related current of $\left(\mathrm{Ba}_{0.4} \mathrm{Sr}_{0.6}\right) \mathrm{TiO}_{3}$ thin films. J. of Applied Physics, 1997, 81(10), 6762-6766.

13. Dietz G.W., Schumacker M., Waser R. et.al. Leakage currents in $\mathrm{Ba}_{0.75} \mathrm{Sr}_{0.25} \mathrm{TiO}_{3}$ thin films for ultrahigh-density dynamic random access memories. J. Appl. Phys., 1997, 82(5), pp. 2359-2364.

14. Wouters D.J., Williams G.J., Herman E. et al. Electrical Conductivity in Ferroelectric Thin Films. J. Microelectr. Engineer., 1995, 29, 249-256.

15. Zafar S., Jones R. E., Jiang Bo., et al. The electronic conduction mechanism in barium strontium titanate thin films. Appl. Phys. Lett., 1998, 73(24), 3533-3535.

16. Stolichnov I. and Tagantsev A. Space-Charge influenced-injection model for conduction in $\mathrm{Pb}\left(\mathrm{Zr}_{x} \mathrm{Ti}_{1-x}\right) \mathrm{O}_{3}$ thin films. J. Appl. Phys., 1998, 84(6), 3216-3225.

17. Sze S.M. Current Transport and Maximum Dielectric Strength of Silicon Nitride Films. J. Appl. Phys., 1967, 38, 2951-2956.

18. Sze S.M., Coleman D.J. Jr and Loya A. Current transport in Metal-Semiconductor-Metal (MMS) structures. Solid State Electronics, 1971, 14, 1209-1218.

19. Sze S.M., Kwok K.Ng. Physics of Semiconductor Devices. A John Wiley \& Sons, Inc., Publ., 2007, 816 p.
20. Gilbert S.R., Wills L.A., Wessels B.W. at al. Electrical transport properties of epitaxial $\mathrm{BaTiO}_{3}$ thin films. J. Appl. Phys., 1996, 80, 969-977.

21. Raymond M.V. and Smyth D.M. Defects and charge transport in perovskite ferroelectrics. J. Phys. Chem Solids, 1996, 57(10), 1507-1511.

22. Gevorgian S.Sh. Ferroelectrics in Microwave Devices, Circuits and Systems. Springer-Verlag, London, 2009, $394 \mathrm{p}$.

23. Tagantsev A.K. and Gerra G. Interfaceinduced phenomena in polarization response of ferroelectric thin films. J. Appl. Phys., 2006, 100, 052607-1-28.

24. Wang R.-V., Mclntyre P.C. Point defect distributions and their electrical effects on (Ba, Sr)TiO/Pt thin films. J. of Applied Physics, 2003, 94(3), 1926-1932.

25. Feil W.A. and Wessels B.W. Defect structure of strontium titanate thin films. J. Appl. Phys., 1993, 74(6), 3927-3931.

26. Park C.H., Scott J., Gerblinger J. et al. Point defect distributions and their electrical effects on (Ba, $\mathrm{Sr}) \mathrm{TiO}_{3} / \mathrm{Pt}(\mathrm{Ba}, \mathrm{Sr}) \mathrm{TiO}_{3} / \mathrm{Pt}$ thin films, J. Appl. Phys., 2003, 94, 1926-1932.

27. Ongaro R., Pillonnet A. Poole-Frenkel (PF) effect high field saturation. Revue de Physique Appliquee, 1989, 24, 1085-1095.

28. Mitrofanov O., Manfa and M. Pool-Frenkel electron emission from the traps in $\mathrm{AlGaN} / \mathrm{GaN}$ transistors. J. of Applied Physics, 2004, 95, 6414-6419.

29. Antula J. Hot-electron concept for PooleFrenkel conduction in amorphous dielectric solids. J. Appl. Phys., 1972, 94(11), 4663-4668.

30. Dawber M., Raba J.F., Scott J.F. Physics of thin-film ferroelectric oxides. Rev. of Modern Phys., 2005, 77, 1083-1130.

31. Gerblinger J., Meixner H. Electrical conductivity of sputtered films of strontium titanate. J. of Applied Physics, 1990, 67, 7453-7459.

32. Vendik O.G., Zubko S.P., Nikol'ski M.A. Microwave loss-factor of $\mathrm{Ba}_{x} \mathrm{Sr}_{1-x} \mathrm{TiO}_{3}$ as a function of temperature, biasing field, barium concentration and frequency. J. of Applied Physics, 2002, 92 (12), 7448-7452.

33. Park C.H., Chadi D.J. Microscopic study of oxygen-vacancy defects in ferroelectric perovskites. Phys. Rev. B, 1998, 57(22), 13961-13964.

34. Buniatyan V., Martirosyan N., Vorobiev A. et al. Dielectric model of point charge defects in insulating paraelectric perovskites. J. of Applied Physics, 2011, 110, 094110-1-11. 
35. Robertson J. Energy levels of point defects in $\mathrm{Ba}(\mathrm{Sr}) \mathrm{TiO}_{3}$ and related oxides. J. of Applied Physics, 2003, 93(2), 1054-1059.

36. Delerue C., Lannoo M., and Allan G. New theoretical approach of transition-metal impurities in semiconductors. Phys. Rev. B39, 1989, 1669-1673.

37. Newille R.C., Hoencisen B. Anomalous capacitance of Schottky barriers on strontium titanate. J. Appl. Phys., 1975, 46(1), 350-353.

38. Wang R.-V., Mclntyre P.C. Point defect distributions and their electrical effects on (Ba, Sr)TiO/Pt thin films. $J$ Appl. Phys., 2003, 94(3), 1926-1932.

39. Zhou C. and Newns D.M. Intrinsic dead effect and the performance of ferroelectric thin film capacitors. J. Appl. Phys., 1997, 82, 30811-388.

40. Cowley A.W. and Sze S.M. Surface States and Barrier Height of Metal-Semiconductor Systems, J. Appl. Phys., 1965, 36, 3212-3218.
41. Warren W.L., Pike G.E., Tuttle B.A. et al. Polarization - induced trapped charge in ferroelectrics, J. Appl. Lett., 1997, 70(15), 2010-2012.

42. Monch W. Role of virtual gap states and defects in metal-semiconductor contacts. Phys. Rev. Lett., 1986, 58, 1260-1268.

43. Copel M., Duncombe P.R., Neumayer D.A. et al. Metallization induced band bending of $\mathrm{SrTiO}_{3}(100) \quad \mathrm{SrTiO}_{3}(100)$ and $\mathrm{Ba}_{0.7} \mathrm{Sr}_{0.3} \mathrm{TiO}_{3}$. Appl. Phys. Lett., 1997, 70, 3227.

44. Chan N.-H., Sharma R.K., and Smyth D.M. Nonstoichiometry in Undoped $\mathrm{BaTiO}_{3}$. J. Am. Ceram. Soc., 1981, 64, 555-562.

45. Nowotny J., Rekas M. Defect Structure, Electrical properties and transport in barium titanate. V. Work Function Ceramics Inter., 1994, 20, 251-255.

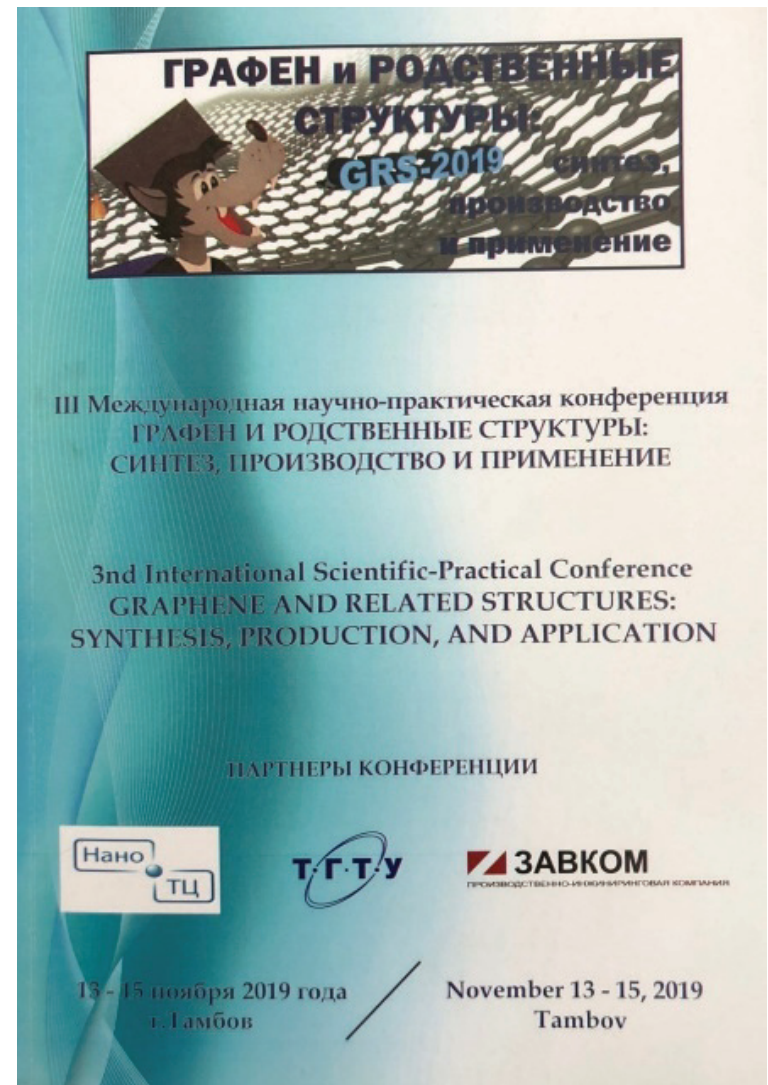

The volume of proceedings contains the papers of the 3rd International Scientific and Practical Conference "GRAPHENE AND RELATED STRUCTURES: SYNTHESIS, PRODUCTION AND APPLICATION" describing the findings of studies in the field of industrial production, applied research and the use of carbon nanomaterials graphene, nanographite, carbon nanotubes and nanofibers, structural materials and functional purpose materials, as well as metrology of standardization and minotoring of carbon nanoproducts. 